\title{
Implant-related MRI artifacts of determined interbody test spacers: artifact calculations due to implant parameters in a porcine spine model
}

\author{
Thorsten Ernstberger \\ Clinic for Spinal Surgery, Klinikum Bad Bramstedt, Bad Bramstedt, Germany; ernstberger@klinikumbb.de
}

Received 21 September 2009; revised 19 October 2009; accepted 20 October 2009

\begin{abstract}
Aim: Intervertebral spacers for anterior spine fusion are made of different materials, which can affect the post-fusion MRI scans. Susceptibility artifacts specially for implants made of titanium alloys can decrease the image quality. This study focused on the influence of determined implant parameters like shape and implant volume in MRI artifacting independent from selected MRI-sequences. Methods: In this study the post-implantation MRI scans of determined cuboids and cylinders were evaluated. All interbody test implants were made of titanium alloys. MRI scans were carried out by using T1 TSE sequences. The total artifact volume (TAV) of all examined implants were calculated for statistical t-test correlation and implant volume (IV)/TAV-relation. Results: Considering all examined test implants with an increasing implant size the TAV became significant larger $(p<0,001)$ with simultaneous reduction of the respective IV/TAV-relation. According to an intergroup TAVcorrelation for cylinders and cuboids with an equivalent implant volume the cylindric test implants demonstrated a significant smaller artifact range $(p<0,05)$. Conclusions: Based on these results the MRI artifacts of larger test implants were more limited to the to the implant's direct surroundings. In this connection for implants with identical material volumes a cylindric shape demonstrated more advantages considering MRI artifacting than cubic forms.
\end{abstract}

Keywords: Magnetic Resonance Imaging;

Geometrical Test Implants; Susceptibility

Effect; Titanium Interbody Spacer

\section{INTRODUCTION}

In the preoperative diagnostics of spinal diseases, mag- netic resonance imaging (MRI) is used as a standard procedure that can visualize disc pathologies and neurological changes of the spinal canal with high precision. When anterior spine fusion proves indicated, implantation of interbody spacers often represents the treatment of choice. Interbody spacers made of various materials can be used as stand-alone cages for exclusively anterior fusion or in combination with dorsal instrumentation for dorsoventral fusion.

When postoperative complications arise secondary to vertebra fusion, MRI scans are frequently necessary to evaluate implant position and demonstrate any clinically relevant abnormalities and to direct further surgical decision-making [1]. However, implant-related susceptibility artifacts can negatively impact the complex post-fusion evaluation of MRI scans. Depending on the spacer material, a local magnetic field gradient of varying susceptibility results in the area between structures. In these border areas, the respective spins gyrate with different frequencies and cause image distortions and susceptibility artifacts [2-4].

The MRI imaging behavior of metallic spinal implants is well documented in the literature [5-11]. However, the aims of the published studies differed in that most focused on determining sequence-related artifact size.

Studies on metallic artifacts in MRI of the anterior thoracic and lumbar spine have been conducted by Vaccaro $e t$ al. [11] and Wang et al. [12]. In one cadaveric study, Vaccaro et al. [11] examined the MRI artifact rates of different metal particles introduced in predefined intervertebral drill holes and subsequently embedded in paraffin. Vaccarro could not demonstrate any significant artifacts in T1- or T2 SE sequences, probably due to the fact that the particle density was lower than that produced by metal implants commonly used in clinical practice.

In another cadaveric artifact study, Wang et al. [12] described the MRI behavior of an intervertebral spacer made of titanium. Using T1 SE sequences, the implantrelated artifact rate of the titanium spacer was primarily limited to the implant's direct surroundings and anatomic neighboring structures were clearly distinguishable. In a phantom study by Rudisch et al. [7]. the relevance of 
Table 1. Test implant's parameters and artifact calculations.

\begin{tabular}{|c|c|c|c|c|c|}
\hline & $\begin{array}{l}\text { Implant parameters } \\
\text { Cylinder: } \\
\text { Height } x \text { base diameter } \\
\text { Cuboid: } \\
\text { Height } x \text { depth } x \text { width } \\
\text { (cm) }\end{array}$ & $\begin{array}{c}\text { Implant volume } \\
\text { (IV) } \\
\left(\mathrm{cm}^{3}\right)\end{array}$ & $\begin{array}{c}\text { Total artifact vol- } \\
\text { ume } \\
\text { (TAV) } \\
( \pm \text { s.d. }) \\
\left(\mathrm{cm}^{3}\right)\end{array}$ & $\begin{array}{c}\text { TAV } \\
\text { p-value }\end{array}$ & $\begin{array}{l}\text { Relation } \\
\text { (IV / TAV) }\end{array}$ \\
\hline \multirow{3}{*}{ 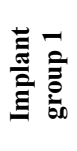 } & Small: $1,5 \times 1,0 \times 1,0$ & 1,5 & $7,0 \quad( \pm 0,14)$ & & $1: 4,7$ \\
\hline & Medium: $2,0 \times 1,2 \times 1,2$ & 2,9 & $11,2 \quad( \pm 0,11)$ & $\mathrm{p}<0,001$ & $1: 3,9$ \\
\hline & Large: $2,5 \times 1,4 \times 1,4$ & 4,9 & $15,2 \quad( \pm 0,16)$ & & $1: 3,1$ \\
\hline \multirow{3}{*}{ 䓂瓷 } & Small: $1,5 \times 1,0$ & 1 & $6,2 \quad( \pm 0,19)$ & & $1: 5,2$ \\
\hline & Medium: 2,0 x 1,2 & 2,3 & $9,1 \quad( \pm 0,23)$ & $\mathrm{p}<0,001$ & $1: 3,9$ \\
\hline & Large: $2,5 \times 1,4$ & 3,9 & $11,4( \pm 0,11)$ & & $1: 2,9$ \\
\hline \multirow{3}{*}{ 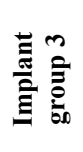 } & Small: $1,91 \times 1,0$ & 1,5 & $6,5 \quad( \pm 0,23)$ & & $1: 4,3$ \\
\hline & Medium: 2,55 x 1,2 & 2,9 & $9,7 \quad( \pm 0,23)$ & $\mathrm{p}<0,001$ & $1: 3,4$ \\
\hline & Large: $3,18 \times 1,4$ & 4,9 & $14,3 \quad( \pm 0,26)$ & & $1: 2,9$ \\
\hline
\end{tabular}

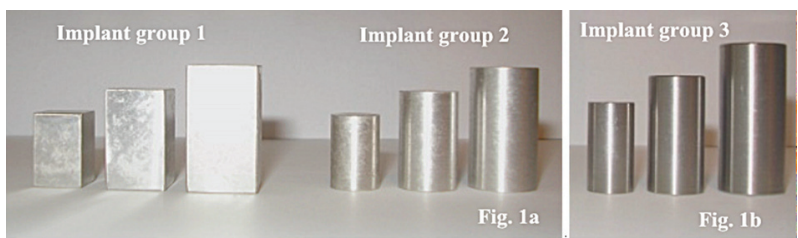

a: Cuboids and cylinders with equivalent heights (Implant group $1+2)$. b: Cylinders with equivalent cuboid volumes (Implant group 3).

Figure 1. $a+b$ : Titanium test implants.

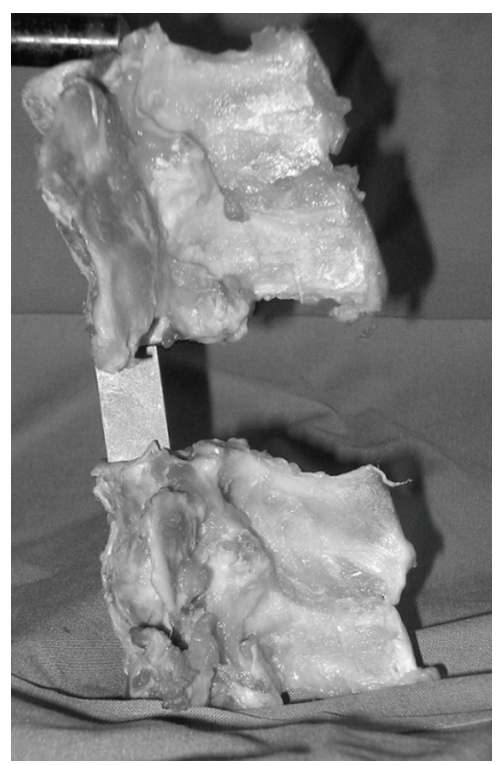

Figure 2. Cadavaric porcine spine model with an implanted large titanium cuboid.

metallic artifacts and implant-related characteristics, such as implant material, shape and position, was demonstrated in addition to an impact by the selected MRI sequence. In spite of the use of optimum MRI sequences, variability in the amount of susceptibility artifacts must be accounted for when evaluating MRI scans of metallic spine implants. This experimental study mainly focused on the influence of the implant parameters shape and volume in MRI artifacting. Therefore in an in vitro spine model, we evaluated the post-implantation MRI scans of determined cubic and cylindric test implants made of titanium alloy. Our hypothesis was that defined implant parameters of geometrical implants would have a clear affect to the range of susceptibility artifacts independent from the selected MRI sequences or used implant materials.

\section{MATERIAL AND METHODS}

In this MRI-study we assessed 3 cuboids (Implant group 1) and 3 cylinders (Implant group 2) (Figure 1a) made of titanium-aluminium-vanadium alloy (TiAL6V4). The test implants of both groups were divided in small, medium and large on account to the implant height. Considering the implant volumes of the cuboids in a third group titanium cylinders with equivalent volumes (Implant group 3) were examined additionally (Figure 1b). The implant parameters of all test implants were listed in Table 1. To visualize differences of the artifact range the respective test implants were exactly placed between adjacent vertebras of a cadaveric Göttingen mini pig spine model (Figure 2). The porcine spine model was completely coated with a soft tissue mass and later stored into a plastic container. For comparable trial conditions markings were drawn to the container wall to reproduce the spine/implant position. After completion of the preparation the MRI investigation followed. For less MRI artifacting MRI scans were carried out by using T1 TSE sequences $[13,14]$.

\subsection{Magnetic Resonance Imaging}

MRI was performed with a $1.5 \mathrm{~T}$ MRI (Magnetom- 
Table 2. TAV inter group correlation of implant group $1+3$.

\begin{tabular}{|c|c|c|c|}
\hline $\begin{array}{l}\text { Implant } \\
\text { group }\end{array}$ & $\begin{array}{l}\text { Implant volume } \\
\qquad\left(\mathrm{cm}^{3}\right)\end{array}$ & $\begin{array}{c}\text { Total artifact volume } \\
\text { (TAV) } \\
( \pm \text { s.d. }) \\
\left(\mathrm{cm}^{3}\right)\end{array}$ & $\begin{array}{c}\text { TAV } \\
\text { (t-test) } \\
\text { p-value }\end{array}$ \\
\hline $\begin{array}{l}1 \\
3\end{array}$ & Small: 1,5 & $\begin{array}{ll}7,0 & ( \pm 0,14) \\
6,5 & ( \pm 0,23)\end{array}$ & 0,027 \\
\hline $\begin{array}{l}1 \\
3\end{array}$ & Medium: 2,9 & $\begin{array}{cc}11,2 & ( \pm 0,11) \\
9,7 & (+0,23)\end{array}$ & 0,002 \\
\hline $\begin{array}{l}1 \\
3\end{array}$ & Large: 4,9 & $\begin{array}{ll}15,2 & ( \pm 0,16) \\
14,3 & ( \pm 0,26)\end{array}$ & 0,006 \\
\hline
\end{tabular}

Symphony,Siemens AG Medical Solutions, Erlangen, Germany. T1w-TSE sequences (TR: $600+2260$, TE: 14 , Flip angle: 15, Band width: 150) were used to acquire a slice thickness of $3 \mathrm{~mm}$ (Figure 3a-c). We selected a matrix with 512 × 512 combined with a Field of View (FOV) of $500 \mathrm{mms}$.

All MRI scans were evaluated 5 times to determine the implant-related TAV by using an actual version of the DICOM reader software. Based on the multisection slice technique for cardiovolumetric MRI analysis (15), the respective TAV were calculated. The artifact area of every MRI slice were determined and later added regarding a slice thickness of $3 \mathrm{~mm}$. Additionally we cal-culated the implant volume/TAV-relation.

\subsection{Statistical Analysis}

Considering a reliable analysis the TAV as well as implant volume (IV)/ TAV-relation of the cuboids and cylinders were determined. To calculate significant differences of the respective TAV within every implant group NewmanKeuls multiple comparisons were carried out (Table 1). Additionally the TAV of cylindric and cubic test implants with an equivalent implant volume (Implant group 1+3) were tested for statistical t-test correlation (Table 2).

\section{RESULTS}

Concerning the repeated artifact measurements for each implant group at the 0,05 significans level no significant differences could be demonstrated. Independent from the implant shape with an increasing implant size the TAV became significant larger $(\mathrm{p}<0,001)$ with simultaneous reduction of the IV/TAV relation within the respective implant group (Table 1). In this context with an increasing implant size the artifact range were more limited to the implant's surrounding. Considering an intergroup TAV correlation a statistical significance between cuboids and cylinders with equivalent implant volumes (Implant group $1+3)$ could be demonstrated $(p<0,05)$ (Table 2). All cylinders of implant group 3 represented a significant smaller artifact range than the correponding cubic test implants of group 1. In this connection for implants with identical material volumes a cylindric shape demonstrated more advantages considering MRI artifacting than cubic forms. Additional correlations did not result in

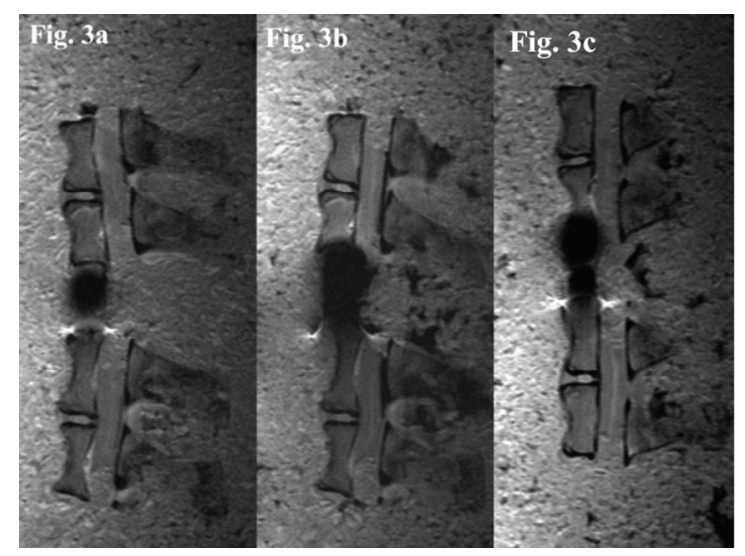

a: Cylinder (small).

b: Cuboid (large).

c: Cylinder (medium)with equivalent cuboid volume.

Figure 3. a-c: Median MRI artifact range depicted in a selection of 3 test implants.

further information.

\section{DISCUSSIONS}

The disadvantages associated with bone grafting alone has led to the development of interbody spacers to enhance anterior spinal fusion $[16,17]$. The use of interbody spacers of different designs and materials has thus become increasingly widespread in clinical routine because they offering immediate load transmission with direct primary stability. In this context the implant design of many interbody spacers derived from a cylindrical or cubic prototype.

In our department, MRI is the radiological diagnostic method of choice for clarifying post-fusion questions regarding the involvement of osseous and soft tissue structures in relation to implant position. In these indications, MRI is better suited than multisection CT to demonstrate myelopathies, inflammatory and infectious pro cesses and any neurodegenerative changes. When postoperative complications arise secondary to vertebra fusion, MRI scans are frequently necessary to demonstrate any clinically relevant abnormalities and to direct further surgical decision-making [4].

The MRI imaging behavior of spinal implants has been widely studied [5-12]. However, the aims of the 
published studies differed in that most focused on determining sequence-related artifact size.

In spite of the use of optimum MRI sequences, variability in the amount of susceptibility artifacts must be accounted for when evaluating MRI scans of metallic spine implants.

Ernstberger et al [18] evaluated in an in vitro study the post-implantation MRI scans of 3 intervertebral disc spacers that differed in shape, material (Titanium, Carbon, Cobalt-chromium), surface qualities and implantation technique. A spacer made of human cortical bone was used as a control. The respective artifactaffected image quality was rated on a developed score. Turbo spin echo sequences produced the best scores for all spacers and the control. Only the control achieved a score of $100 \%$. The carbon, titanium and cobalt-chrome spacers scored $83.3 \%, 62.5 \%$ and $50 \%$, respectively.

In this study geometrical implant forms like cylinders and cuboids were chosen to prove possible coherences between the range of MRI artifacting and determined implant related factors like shape and implant volume. For the cuboids as well as cylinders the range of MRI artifacts was directly affected by the implant size and volume. In this context the smaller the implant the smaller the range of susceptibility artifacts. For implants characterized by equivalent materials and implant volumes the influence of the implant shape on the artifact size has to be proofed with priority on an artifact volumetric analysis. In this context significant differences could be determined in favor of a cylindric implant shape. For all examined implant groups a more advantageous IV/TAV-relation could be recognized the larger the implants. In this connection using T1 TSE sequences, the expected implant-related artifact rate are more limited to the implant's direct surroundings. On the basis of our study results the range of artifacts of our used test implants were influenced by material volume as well as implant shape. Considering the implant design of current intervertebral spacers further studies are neccessary to interpreted the influence and concurrency of implant characteristics in MRI artifacting.

\section{REFERENCES}

[1] J. W. Van Goethem, P. M. Parizel, J. R. Jinkins, (2002) Review article: MRI of the postoperative lumbar spine. Neuroradiology, 44, 723-39.

[2] C. Fellner, M. Behr, F. Fellner, P. Held, G. Handel, S. Feuerbach, (1997). Artifacts in MR imaging of the temporomandibular joint caused by dental alloys: A phantom model study at T1.5. Rofo, $\mathbf{1 6 6}, 421-8$.

[3] S. Fritzsche, R. Thull, A. Haase, (1994) Reduction of artifacts in magnetic resonance images by using optimized materials for diagnostic devices and implants. Biomed
Tech (Berl), 39, 42-6.

[4] J. F. Schenck, (1996) The role of magnetic susceptibility in magnetic resonance imaging: MRI magnetic compatibility of the first and second kinds. Med Phys, 23, 815-50.

[5] A. S. Malik, O. Boyko, N. Atkar, W. F. Young, (2001) A comparative study of MR imaging profile of titanium pedicle screws. Acta Radiol, 42, 291-3.

[6] C. A. Petersilge, J. S. Lewin, J. L. Duerk, J. U. Yoo, A. J. Ghaneyem, (1996) Optimizing imaging parameters for MR evaluation of the spine with titanium pedicle screws. AJR Am J Roentenol, 166, 1213-8.

[7] A. Rudisch, C. Kremser, S. Peer, A. Kathrein, W. Judmaier, H. Daniaux, (1998) Metallic artifacts in magnetic resonace imaging of patients with spinal fusion. A comparison of implant materials and implant sequences. Spine, 23, 692-9.

[8] R. Rupp, N. A. Ebraheim, E. R. Savolaine, W. T. Jackson, (1993) Magnetic resonance imaging evaluation of the spine with metal implants. General safety and superior imaging with titanium. Spine, 18, 379-85.

[9] M. Thomsen, U. Schneider, S. J. Breusch, J. Hansmann, M. Freund, (2001) Artefacts and ferromagnetism dependent on different metal alloys in magnetic resonance imaging. An experimental study. Orthopade, 30, 40-4.

[10] J. C. Wang, H. S. Sandhu, M. D. Yu, J. T. Minchew, R. B. Delamarter, (1997) MR parameters for imaging titanium spinal instrumentation. J Spinal Disord, 10, 27-32

[11] A. R. Vaccaro, R. M. Chesnut, G. Scuderi, J. F. Healy, J. B. Massie, S. R. Garfin, (1994) Metallic spinal artifacts in magnetic resonance imaging. Spine, 19, 1237-42.

[12] J. C. Wang, W. D. Yu, H. S. Sandhu, V. Tam, R. B. Delamarter, (1998) A comparison of magnetic resonance and computed tomographic image quality after the implantation of tantalum and titanium spinal instrumentation. Spine, 23, 1684-8.

[13] C. B. Henk, W. Brodner, S. Grampp, M. Breitenseher, M. Thurnher, G. H. Mostbeck, H. Imhof, (1999) The postoperative spine. Top Magn Reson Imaging, 10, 247-64.

[14] T. Herold, W. C. Caro, G. Heers, L. Perlick, J. Grifka, S. Feuerbach, W. Nitz, M .Lenhart, (2004) Influence of sequence type on the extent of the susceptility artifact in MRIa shoulder specimen study after suture anchor repair. Rofo, 176, 1296-301.

[15] J. F. Debatin, S. N. Nadel, H. D. Sostman, C. E. Spritzer, A. J. Evans, T. M. Grist, (1992) Magnetic resonance imaging cardiac ejection fraction measurements. Phantom study comparing four different methods. Invest Radiol, 27, 198204.

[16] B. N. Summers and S. M. Eisenstein, (1989) Donor site pain from the ilium. A complication of lumbar spine fusion. J Bone Joint Surg Br, 71, 677-80.

[17] J. A. Goulet, L. E. Senunas, G. L. DeSilva, M. L. Greenfield, (1997) Autogenous iliac crest bone graft. Complications and functional assessment. Clin Orthop, 339, 76-81.

[18] T. Ernstberger, G. Heidrich, T. Bruning, S. Krefft, G. Buchhorn, H. M. Klinger, (2007) The inter-observer validated relevance of intervertebral spacer materials in MRI artifacting. Eur Spine J, 16, 179-85. 